\title{
On sums of Szemerédi-Trotter sets *
}

\author{
Shkredov I.D.
}

\begin{abstract}
Annotation.
We prove new general results on sumsets of sets having Szemerédi-Trotter type. This family includes convex sets, sets with small multiplicative doubling, images of sets under convex/concave maps and others.
\end{abstract}

\section{Introduction}

Let $A=\left\{a_{1}, \ldots, a_{n}\right\}, a_{i}<a_{i+1}$ be a set of real numbers. We say that $A$ is convex if

$$
a_{i+1}-a_{i}>a_{i}-a_{i-1}
$$

for every $i=2, \ldots, n-1$. Lower bounds for sumsets/difference sets of convex sets were obtained in several papers, see [4], [1], [2], [3], [5], [16], [13, [8], [1] and others. For example, in [8] the following theorem was proved.

Theorem 1 Let $A \subset \mathbb{R}$ be a convex set. Then

$$
|A+A| \gg|A|^{\frac{14}{9}} \log ^{-\frac{2}{9}}|A| .
$$

In our paper we obtain a series of results on sumsets/difference sets of rather general families of sets, including convex sets, see Theorems 11, 14 below. In particular, it allows us to refine the result above.

Theorem 2 Let $A \subset \mathbb{R}$ be a convex set. Then

$$
|A+A| \gg|A|^{\frac{58}{37}} \log ^{-\frac{20}{37}}|A| .
$$

Moreover, our method gives a generalization of Theorem 1 for sumset of two different convex sets, see Theorem 14 below.

In 9] the authors prove a general statement on addition of a set and its image under a convex map (the first result in the direction was obtained in [3]).

\footnotetext{
${ }^{*}$ This work was supported by grant Russian Scientific Foundation RSF 14-11-00433.
} 
Theorem 3 Let $f$ be any continuous, strictly convex or concave function on the reals, and $A, C \subset \mathbb{R}$ be any finite sets such that $|A|=|C|$. Then

$$
|f(A)+C|^{10}|A+A|^{9} \gg|A|^{24} \log ^{-2}|A| .
$$

In particular, choosing $C=f(A)$, we get

$$
\max \{|f(A)+f(A)|,|A+A|\} \gg|A|^{\frac{24}{19}} \log ^{-\frac{2}{19}}|A| .
$$

Finally

$$
|A A|^{10}|A+A|^{9} \gg|A|^{24} \log ^{-2}|A|
$$

We refine the result.

Theorem 4 Let $f$ be any continuous, strictly convex or concave function on the reals, and $A, C \subset \mathbb{R}$ be any finite sets such that $|A|=|C|$. Then

$$
|f(A)+C|^{42}|A+A|^{37} \gg|A|^{100} \log ^{-20}|A| .
$$

In particular, choosing $C=f(A)$, we get

$$
\max \{|f(A)+f(A)|,|A+A|\} \gg|A|^{\frac{100}{79}} \log ^{-\frac{20}{79}}|A| .
$$

Finally

$$
|A A|^{42}|A+A|^{37} \gg|A|^{100} \log ^{-20}|A|
$$

Another applications can be found in the last section 4 .

In the proof we use so-called the eigenvalues method, see e.g. [15] and some observations from [14].

\section{Notation}

Let $\mathbf{G}$ be an abelian group and + be the group operation. In the paper we use the same letter to denote a set $S \subseteq \mathbf{G}$ and its characteristic function $S: \mathbf{G} \rightarrow\{0,1\}$. By $|S|$ denote the cardinality of $S$.

Let $f, g: \mathbf{G} \rightarrow \mathbb{C}$ be two functions with finite supports. Put

$$
(f * g)(x):=\sum_{y \in \mathbf{G}} f(y) g(x-y) \quad \text { and } \quad(f \circ g)(x):=\sum_{y \in \mathbf{G}} f(y) g(y+x)
$$

Let $A_{1}, \ldots, A_{k} \subseteq \mathbf{G}$ be any sets. Put

$$
\mathrm{E}_{k}\left(A_{1}, \ldots, A_{k}\right)=\sum_{x \in \mathbf{G}}\left(A_{1} \circ A_{1}\right)(x) \ldots\left(A_{k} \circ A_{k}\right)(x)
$$


be the higher energy of $A_{1}, \ldots, A_{k}$. If $A_{j}=A, j=1, \ldots, k$ we simply write $\mathrm{E}_{k}(A)$ instead of $\mathrm{E}_{k}(A, \ldots, A)$. In the same way one can define $\mathrm{E}_{k}(A)$ for non-integer $k$. In particular case $k=2$ we put $\mathrm{E}(A, B):=\mathrm{E}_{2}(A, B)$ and $\mathrm{E}(A)=\mathrm{E}_{2}(A)$. The quantity $\mathrm{E}(A)$ is called the additive energy of a set, see e.g. [17]. Similarly, we define

$$
\mathrm{E}_{k}\left(f_{1}, \ldots, f_{k}\right)=\sum_{x}\left(f_{1} \circ f_{1}\right)(x) \ldots\left(f_{k} \circ f_{k}\right)(x) .
$$

Denote by $\mathcal{C}_{k+1}\left(f_{1}, \ldots, f_{k+1}\right)\left(x_{1}, \ldots, x_{k}\right)$ the function

$$
\mathcal{C}_{k+1}\left(f_{1}, \ldots, f_{k+1}\right)\left(x_{1}, \ldots, x_{k}\right)=\sum_{z} f_{1}(z) f_{2}\left(z+x_{1}\right) \ldots f_{k+1}\left(z+x_{k}\right) .
$$

Thus, $\mathcal{C}_{2}\left(f_{1}, f_{2}\right)(x)=\left(f_{1} \circ f_{2}\right)(x)$. If $f_{1}=\cdots=f_{k+1}=f$ then write $\mathcal{C}_{k+1}(f)\left(x_{1}, \ldots, x_{k}\right)$ for $\mathcal{C}_{k+1}\left(f_{1}, \ldots, f_{k+1}\right)\left(x_{1}, \ldots, x_{k}\right)$. Note that

$$
\sum_{x_{1}, \ldots, x_{k}} \mathcal{C}_{k+1}^{2}\left(f_{1}, \ldots, f_{k+1}\right)\left(x_{1}, \ldots, x_{k}\right)=\mathrm{E}_{k+1}\left(f_{1}, \ldots, f_{k+1}\right) .
$$

Let $g: \mathbf{G} \rightarrow \mathbb{C}$ be a function, and $A \subseteq \mathbf{G}$ be a finite set. By $\mathrm{T}_{A}^{g}$ denote the matrix with indices in the set $A$

$$
\mathrm{T}_{A}^{g}(x, y)=g(x-y) A(x) A(y) .
$$

It is easy to see that $\mathrm{T}_{A}^{g}$ is hermitian iff $\overline{g(-x)}=g(x)$. The corresponding action of $\mathrm{T}_{A}^{g}$ is

$$
\left\langle\mathrm{T}_{A}^{g} a, b\right\rangle=\sum_{z} g(z)(\bar{b} \circ a)(z) .
$$

for any functions $a, b: A \rightarrow \mathbb{C}$. In the case $\overline{g(-x)}=g(x)$ by $\operatorname{Spec}\left(\mathrm{T}_{A}^{g}\right)$ we denote the spectrum of the operator $\mathrm{T}_{A}^{g}$

$$
\operatorname{Spec}\left(\mathrm{T}_{A}^{g}\right)=\left\{\mu_{1} \geq \mu_{2} \geq \cdots \geq \mu_{|A|}\right\} .
$$

Write $\{f\}_{\alpha}, \alpha \in[|A|]$ for the corresponding eigenfunctions. We call $\mu_{1}$ as the main eigenvalue and $f_{1}$ as the main function.

In the asymmetric case let $g: \mathbf{G} \rightarrow \mathbb{C}$ be a function, and $A, B \subseteq \mathbf{G}$ be two finite sets. Suppose that $|B| \leq|A|$. By $\mathrm{T}_{A, B}^{g}$ denote the rectangular matrix

$$
\mathrm{T}_{A, B}^{g}(x, y)=g(x-y) A(x) B(y),
$$

and by $\tilde{\mathrm{T}}_{A, B}^{g}(x, y)$ denote the another rectangular matrix

$$
\tilde{\mathrm{T}}_{A, B}^{g}(x, y)=g(x+y) A(x) B(y) .
$$

As in (6), we arrange the singular values in order of magnitude

$$
\begin{gathered}
\lambda_{1}\left(\mathrm{~T}_{A, B}^{g}\right) \geq \lambda_{2}\left(\mathrm{~T}_{A, B}^{g}\right) \geq \cdots \geq \lambda_{|B|}\left(\mathrm{T}_{A, B}^{g}\right) \geq 0, \\
\mathrm{~T}_{A, B}^{g}(x, y)=\sum_{j=1}^{|B|} \lambda_{j} u_{j}(x) v_{j}(y)
\end{gathered}
$$

and similar for $\tilde{\mathrm{T}}_{A, B}^{g}$. Here $u_{j}(x), v_{j}(y)$ are singularfunctions of the operators. General theory of such operators was developed in [15].

All logarithms are base 2 . Signs $\ll$ and $\gg$ are the usual Vinogradov's symbols. 


\section{The main definition}

We begin with a rather general definition of families of sets which are usually obtained by Szemerédi-Trotter's theorem, see [17].

Definition $5 A$ set $A \subset \mathbb{R}$ has SzT-type (in other words $A$ is called Szemerédi-Trotter set) with parameter $\alpha \geq 1$ if for any set $B \subset \mathbb{R}$ and an arbitrary $\tau \geq 1$ one has

$$
|\{x \in A+B:(A * B)(x) \geq \tau\}| \ll c(A)|B|^{\alpha} \cdot \tau^{-3},
$$

where $c(A)>0$ is a constant depends on the set $A$ only. We define the quantity $c(A)|B|^{\alpha}$ as $c(A, B)$.

From the definition one can see that if $A$ has SzT-type then $(-A)$ has the same SzT-type with the same parameters $\alpha$ and $c(A)$.

Remark 6 We put parameter $\alpha \geq 1$ because otherwise there is no any SzT-type set. Indeed, take $B=C-A$, where set $C$ will be chosen later. One has $(A * B)(x) \geq|A|$ for any $x \in C$. Then by (9), we obtain

$$
|A|^{3}|C| \ll c(A)|A+C|^{\alpha} \leq c(A)|A|^{\alpha}|C|^{\alpha}
$$

Taking $|C|$ sufficiently large and having the set $A$ is fixed, we see that $\alpha \geq 1$.

Examples. Let us give some examples of SzT-type sets with parameter $\alpha=2$.

1) If $A \subset \mathbb{R}$ is a convex set then $A$ has SzT-type with $c(A)=|A|$, see [13.

2) Let $f$ be a strictly convex/concave function. Then $f(A)$ has SzT-type with $c(A)=q(A)$, where

$$
q(A):=\min _{C} \frac{|A+C|^{2}}{|C|},
$$

and $A$ has SzT-type with $c(A)=q(f(A))$, see [12, [9].

$3)$ Let $|A A| \leq M|A|$. Then $A$ has SzT-type with $c(A)=M^{2}|A|$. This is a particular case of the family from (2). Indeed, take $f(x)=\log x$, and apply (10) with $C=\log \left(A \cap \mathbb{R}^{+}\right)$or $C=\log \left|\left(A \cap \mathbb{R}^{-}\right)\right|$.

4) Let $A \subset \mathbb{R}^{+}$, and $a \in \mathbb{R} \backslash\{0\}$. Then $\log A$ has SzT-type with $c(A)=q^{\prime}(A)$, where

$$
q^{\prime}(A):=\min _{C} \frac{|(A+a) C|^{2}}{|C|},
$$

see [6], [10].

There are another families of SzT-type sets, for example see a family of complex sets in [7].

Using definition 5 and easy calculations, one can obtain upper bounds for some simple characteristics of SzT-type sets see, e.g. papers [12, [13, 8], 9]. It is more convenient do not use parameter $\alpha$ in the statements. 
Lemma 7 Let $A$ be a SzT-type set. Then

$$
\mathrm{E}_{3}(A) \ll c(A, A) \log |A|, \quad \mathrm{E}^{3}(A) \ll \mathrm{E}_{3 / 2}^{2}(A) c(A, A),
$$

and for any $B$ one has

$$
\mathrm{E}(A, B) \ll(c(A, B)|A||B|)^{1 / 2} .
$$

We need in one more technical lemma.

Lemma 8 Let $A, A_{*}$ be a SzT-type set with the same parameter $\alpha>1$. Then

$$
\mathrm{E}^{2 \alpha-1}\left(A_{*}, A\right) \ll_{(\alpha-1)^{-1}}\left(\sum_{x}\left(A_{*} \circ A_{*}\right)^{1 / 2}(x)(A \circ A)(x)\right)^{2 \alpha-2} \cdot c^{1 / 3}(A) c^{\alpha / 3}\left(A_{*}\right)|A|^{2 / 3}\left|A_{*}\right|^{\alpha^{2} / 3} .
$$

Proof. Put $c_{*}=c\left(A_{*}\right), c=c(A), a=|A|, a_{*}=\left|A_{*}\right|$. Splitting the sum, we get with some inaccuracy

$$
\mathrm{E}\left(A_{*}, A\right) \ll \tau^{1 / 2}\left(\sum_{x}\left(A_{*} \circ A_{*}\right)^{1 / 2}(x)(A \circ A)(x)\right)+\tau \sum_{x} S_{\tau}(x)(A \circ A)(x)=\tau^{1 / 2} \omega_{1}+\tau \omega_{2},
$$

where $S_{\tau}=\left\{x:\left(A_{*} \circ A_{*}\right)(x) \geq \tau\right\}$. Because of $A_{*}$ is Szemerédi-Trotter set, we have $\left|S_{\tau}\right| \ll$ $c_{*}\left(a_{*}\right)^{\alpha} \tau^{-3}$. On the other hand, $A$ is also Szemerédi-Trotter set, so

$$
\sum_{x} S_{\tau}(x)(A \circ A)(x)=\sum_{x \in A}\left(S_{\tau} * A\right)(x) \ll c^{1 / 3}\left|S_{\tau}\right|^{\alpha / 3} a^{2 / 3} \ll c^{1 / 3} a^{2 / 3} c_{*}^{\alpha / 3}\left(a_{*}\right)^{\alpha^{2} / 3} \tau^{-\alpha}
$$

Combining (11) and (12), we obtain

$$
\mathrm{E}\left(A_{*}, A\right) \ll_{(\alpha-1)^{-1}} \tau^{1 / 2} \omega_{1}+\tau^{1-\alpha} c^{1 / 3} a^{2 / 3} c_{*}^{\alpha / 3}\left(a_{*}\right)^{\alpha^{2} / 3}
$$

An optimal choice of parameter $\tau$ is $\tau^{1 / 2}=\omega_{1}^{-1 /(2 \alpha-1)} c^{1 / 3(2 \alpha-1)} c_{*}^{\alpha / 3(2 \alpha-1)} a^{2 / 3(2 \alpha-1)} a_{*}^{\alpha^{2} / 3(2 \alpha-1)}$. Thus

$$
\mathrm{E}^{2 \alpha-1}\left(A_{*}, A\right) \ll_{(\alpha-1)^{-1}}\left(\sum_{x}\left(A_{*} \circ A_{*}\right)^{1 / 2}(x)(A \circ A)(x)\right)^{2 \alpha-2} \cdot c^{1 / 3} c_{*}^{\alpha / 3} a^{2 / 3} a_{*}^{\alpha^{2} / 3}
$$

as required. 


\section{The proof of the main result}

We begin with a lemma from [15].

Lemma 9 Let $A \subseteq \mathbf{G}$ be a set and $g$ be a nonnegative function on $\mathbf{G}$. Suppose that $f_{1}$ is the main eigenfunction of $\mathrm{T}_{A}^{g}$ or $\tilde{\mathrm{T}}_{A}^{g}$, and $\mu_{1}$ is the correspondent eigenvalue. Then

$$
\left\langle\mathrm{T}_{A}^{A \circ A} f_{1} f_{1}\right\rangle \geq \frac{\mu_{1}^{3}}{\|g\|_{2}^{2} \cdot\|g\|_{\infty}} .
$$

A particular case $\alpha=2$ of the next lemma is contained inside Theorem 8 of paper [14]. We give the proof for the sake of completeness.

Lemma 10 Let $A$ be a SzT-type set and let $\Delta \geq 1$ be a real number. Suppose that

$$
B \subseteq\{x:(A \circ A)(x) \geq \Delta\} \quad \text { or } \quad B \subseteq\{x:(A * A)(x) \geq \Delta\} .
$$

Then

$$
\mathrm{E}_{3}(A, A, B) \ll \Delta^{-\frac{4}{3 \alpha-1}} c(A)^{\frac{5 \alpha+1}{2(3 \alpha-1)}}|A|^{\frac{2 \alpha^{2}+5 \alpha-1}{2(3 \alpha-1)}}|B|^{\frac{3\left(\alpha^{2}-1\right)}{2(3 \alpha-1)}} \log |A|
$$

Proof. Put $a=|A|, L=\log a$ and $c=c(A)$. By the pigeonhole principle there is a set $Q$ such that

$$
\sigma:=\mathrm{E}_{3}(A, A, B) \ll L \sum_{x \in Q}(A \circ A)^{2}(x)(B \circ B)(x),
$$

and the values of the convolution $(A \circ A)(x)$ differ at most twice on $Q$. Denote by $q$ the maximum of $(A \circ A)(x)$ on $Q$. Because of $A$ is SzT-type set, we have $|Q| \ll c a^{\alpha} q^{-3}$. Using Lemma 7, we obtain

$$
\sigma \ll L q \mathrm{E}(A, B) \ll L q\left(c(A) a|B|^{\alpha+1}\right)^{1 / 2} .
$$

On the other hand, by the definition of the set $B$ and the Cauchy-Schwarz inequality one has

$$
\sigma \ll L q^{2} \Delta^{-1} \mathrm{E}(Q, B, A, A) \ll L q^{2} \Delta^{-1} \mathrm{E}^{1 / 2}(Q, A) \mathrm{E}^{1 / 2}(B, A) .
$$

Combining the last two bounds and the upper bound for size of $Q$, we have

$$
\begin{gathered}
\sigma \ll L q \mathrm{E}^{1 / 2}(A, B)\left(\mathrm{E}^{1 / 2}(A, B)+q \Delta^{-1} c^{1 / 4} a^{1 / 4}|Q|^{(\alpha+1) / 4}\right) \ll \\
\ll L q \mathrm{E}^{1 / 2}(A, B)\left(\mathrm{E}^{1 / 2}(A, B)+q^{-(3 \alpha-1) / 4} \Delta^{-1} c^{(\alpha+2) / 4} a^{\left(\alpha^{2}+\alpha+1\right) / 4}\right) .
\end{gathered}
$$

The optimal choice of $q$ is $q=\mathrm{E}^{-2 /(3 \alpha-1)}(A, B) \Delta^{-4 /(3 \alpha-1)} c^{(\alpha+2) /(3 \alpha-1)} a^{\left(\alpha^{2}+\alpha+1\right) /(3 \alpha-1)}$. Here we have used the fact that $\alpha>1 / 3$. Substituting $q$ into the last formula and using Lemma 7 again, we get

$$
\begin{gathered}
\sigma \ll L q \mathrm{E}(A, B) \ll L \mathrm{E}^{3(\alpha-1) /(3 \alpha-1)}(A, B) \cdot \Delta^{-4 /(3 \alpha-1)} c^{(\alpha+2) /(3 \alpha-1)} a^{\left(\alpha^{2}+\alpha+1\right) /(3 \alpha-1)}= \\
=L \Delta^{-\frac{4}{3 \alpha-1}}|B|^{\frac{3\left(\alpha^{2}-1\right)}{6 \alpha-2}} c^{\frac{5 \alpha+1}{6 \alpha-2}} a^{\frac{2 \alpha^{2}+5 \alpha-1}{6 \alpha-2}}
\end{gathered}
$$

as required.

Let us formulate the main result of the paper. 
Theorem 11 Suppose that $A \subset \mathbb{R}$ has SzT-type with parameter $\alpha$. Then

$$
|A+A| \gg c(A)^{\frac{1-11 \alpha}{3 \alpha^{2}+12 \alpha+1}}|A|^{\frac{-8 \alpha^{2}+57 \alpha-3}{3 \alpha^{2}+12 \alpha+1}} \cdot(\log |A|)^{-\frac{4(3 \alpha-1)}{3 \alpha^{2}+12 \alpha+1}} .
$$

In particular, for $\alpha=2$ one has

$$
|A+A| \gg c(A)^{-\frac{21}{37}}|A|^{\frac{79}{37}} \cdot(\log |A|)^{-\frac{20}{37}} .
$$

Pro of. Let $S=A+A,|S|=d, a=|A|$. Let also $L=\log a, c=c(A)$. We have

$$
|A|^{2}=\sum_{x, y} A(x) A(y) S(x+y) \leq 2 \sum_{z \in S_{1}}(A * A)(z),
$$

where $S_{1}=\left\{z \in S:(A * A)(z) \geq 2^{-1} a^{2} d^{-1}\right\}$. Denote by $f_{j}, \mu_{j}$ the eigenfunctions and eigenvalues of hermitian operator $\tilde{\mathrm{T}}_{A}^{S_{1}}$. From (18) it follows that $\mu_{1} \geq a / 2$. Applying Lemma 9 , we see that

$$
\left\langle\mathrm{T}_{A}^{A \circ A} f_{1}, f_{1}\right\rangle \geq \mu_{1}^{3}\left(\tilde{\mathrm{T}}_{A}^{S_{1}}\right) d^{-1} \geq 2^{-3} a^{3} d^{-1} .
$$

Further, by nonnegativity of the operator $\mathrm{T}_{A}^{A \circ A}$ as well as inequality (19) and the lower bound for $\mu_{1}$, we get

$$
\sigma:=\sum_{x, y, z} \mathrm{~T}_{A}^{A \circ A}(x, y) \tilde{\mathrm{T}}_{A}^{S_{1}}(x, z) \tilde{\mathrm{T}}_{A}^{S_{1}}(y, z)=\sum_{j=1}^{a} \mu_{j}^{2}\left\langle\mathrm{~T}_{A}^{A \circ A} f_{j}, f_{j}\right\rangle \geq \mu_{1}^{2}\left\langle\mathrm{~T}_{A}^{A \circ A} f_{1}, f_{1}\right\rangle \geq 2^{-5} a^{5} d^{-1} .
$$

On the other hand

$\sigma=\sum_{x, y, z \in A}(A \circ A)(x-y) S_{1}(x+z) S_{1}(y+z)=\sum_{\alpha, \beta} S_{1}(\alpha) S_{1}(\beta)(A \circ A)(\alpha-\beta) \mathcal{C}_{3}(-A, A, A)(\alpha, \beta)$.

Combining (20), (21) and using (4), we obtain by the Cauchy-Schwarz inequality that

$$
a^{10} d^{-2} \ll \mathrm{E}_{3}(A) \mathrm{E}_{3}\left(A, A, S_{1}\right) .
$$

Applying the first formula of Lemma 7 to estimate the quantity $\mathrm{E}_{3}(A)$ and Lemma 10 to estimate $\mathrm{E}_{3}\left(A, A, S_{1}\right)$, we have

$$
a^{10} d^{-2} \ll L^{2} a^{\alpha} c \cdot \Delta^{-\frac{4}{3 \alpha-1}} c^{\frac{5 \alpha+1}{2(3 \alpha-1)}} a^{\frac{2 \alpha^{2}+5 \alpha-1}{2(3 \alpha-1)}} d^{\frac{3\left(\alpha^{2}-1\right)}{2(3 \alpha-1)}},
$$

where $\Delta=2^{-1} a^{2} d^{-1}$. After some calculations, we get

$$
d \gg L^{-\frac{4(3 \alpha-1)}{3 \alpha^{2}+12 \alpha+1}} c^{\frac{1-11 \alpha}{3 \alpha^{2}+12 \alpha+1}} a^{\frac{-8 \alpha^{2}+57 \alpha-3}{3 \alpha^{2}+12 \alpha+1}}
$$

as required.

Proof of Theorems 2, 4, To obtain Theorem 2 just recall that $\alpha=2, c(A)=|A|$ for convex sets. Remembering the definition of $q(f(A))$ from (10), we have $c(A)=q(f(A)) \leq$ $|f(A)+C|^{2}|C|^{-1}$. After that applying the main Theorem 11, we get Theorem 4 . 


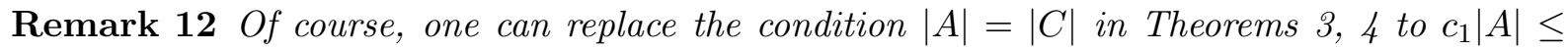
$|C| \leq c_{2}|A|$, where $c_{1}, c_{2}>0$ are any absolute constants. Certainly, signs $\ll, \gg$ should be changed by $\ll_{c_{1}, c_{2}}, \gg_{c_{1}, c_{2}}$ in the case. Even more, it is possible to prove the results for sets $A$ and $C$, having incomparable sizes. We do not make such calculations here (and also below), note only that because in Theorem 4, we have $c(A) \leq|f(A)+C|^{2}|C|^{-1}$ this implies

$$
|f(A)+C|^{42}|A+A|^{37} \gg|A|^{79}|C|^{21} \log ^{-20}|A| .
$$

We conclude the section proving a result which generalize, in particular, Theorem 1.3 from [9] as well as the results on sumsets/difference sets of convex sets from [13]. The arguments are in the spirit of Theorem 11. We need in a lemma from [15].

Lemma 13 Let $A, B \subseteq \mathbf{G}$ be finite sets, $|B| \leq|A|, D, S \subseteq \mathbf{G}$ be two sets such that $A-B \subseteq D$, $A+B \subseteq S$. Then the main eigenvalues and singularfunctions of the operators $\mathrm{T}_{A, B}^{D}, \tilde{\mathrm{T}}_{A, B}^{S}$ equal $\lambda_{1}=(|A||B|)^{1 / 2}$, and

$$
v_{1}(y)=B(y) /|B|^{1 / 2}, \quad \text { and } \quad u_{1}(x)=A(x) /|A|^{1 / 2} .
$$

All other singular values equal zero.

Using lemma above, we prove our second main result, although one can use a more elementary approach as in [13.

Theorem 14 Suppose that $A, A_{*} \subset \mathbb{R}$ have SzT-type with the same parameter $\alpha$. Then

$$
\begin{aligned}
& \left|A \pm A_{*}\right| \gg \\
& \min \left\{c\left(A_{*}\right)^{-\frac{2}{3(7+\alpha)}} c(A)^{-\frac{13}{3(7+\alpha)}}\left|A_{*}\right|^{\frac{2(24-\alpha)}{3(7+\alpha)}}|A|^{\frac{33-10 \alpha}{3(7+\alpha)}}, c(A)^{-\frac{2}{3(7+\alpha)}} c\left(A_{*}\right)^{-\frac{13}{3(7+\alpha)}}|A|^{\frac{2(24-\alpha)}{3(7+\alpha)}}\left|A_{*}\right|^{\frac{33-10 \alpha}{3(7+\alpha)}}\right\} \\
& \times\left(\log \left(|A|\left|A_{*}\right|\right)\right)^{-\frac{2}{7+\alpha}}
\end{aligned}
$$

and for $\alpha>1$

$$
\begin{aligned}
\left|A \pm A_{*}\right| \gg_{(\alpha-1)^{-1}} c(A)^{-\frac{4 \alpha-2}{3\left(\alpha^{2}+4 \alpha-3\right)}} c\left(A_{*}\right)^{-\frac{7 \alpha-5}{3\left(\alpha^{2}+4 \alpha-3\right)}}|A|^{\frac{28 \alpha-4 \alpha^{2}-16}{3\left(\alpha^{2}+4 \alpha-3\right)}}\left|A_{*}\right|^{\frac{35 \alpha-4 \alpha^{2}-21}{3\left(\alpha^{2}+4 \alpha-3\right)}} \\
\times\left(\log \left(|A|\left|A_{*}\right|\right)\right)^{-\frac{2(\alpha-1)}{\alpha^{2}+4 \alpha-3}} .
\end{aligned}
$$

In particular, for $\alpha=2$ one has

$$
\begin{gathered}
\left|A \pm A_{*}\right| \gg \max \left\{c\left(A_{*}\right)^{-\frac{1}{3}} c(A)^{-\frac{2}{9}}\left|A_{*}\right|^{\frac{11}{9}}|A|^{\frac{8}{9}}, c(A)^{-\frac{1}{3}} c\left(A_{*}\right)^{-\frac{2}{9}}|A|^{\frac{11}{9}}\left|A_{*}\right|^{\frac{8}{9}}\right. \\
\left.\min \left\{c\left(A_{*}\right)^{-\frac{2}{27}} c(A)^{-\frac{13}{27}}\left|A_{*}\right|^{\frac{44}{27}}|A|^{\frac{13}{27}}, c(A)^{-\frac{2}{27}} c\left(A_{*}\right)^{-\frac{13}{27}}|A|^{\frac{44}{27}}\left|A_{*}\right|^{\frac{13}{27}}\right\}\right\} \times \\
\times\left(\log \left(|A|\left|A_{*}\right|\right)\right)^{-\frac{2}{9}}
\end{gathered}
$$

Finally

$$
\left|A \pm A_{*}\right|^{\frac{\alpha+1}{2}}|A-A| \gg|A|^{\frac{33-4 \alpha}{6}}\left|A_{*}\right|^{\frac{6-\alpha}{3}} c^{-7 / 6}(A) c^{-1 / 3}\left(A_{*}\right) \log ^{-1}\left(|A|\left|A_{*}\right|\right)
$$


Proof. Because SzT-types of the sets $A$ and $(-A)$ are coincide it is sufficient to prove the result for sums. Let $S=A+A_{*},|S|=d, a=|A|, a_{*}=\left|A_{*}\right|$. Let also $L=\log \left(a a_{*}\right), c=c(A)$, $c_{*}=c\left(A_{*}\right)$. By the Cauchy-Schwarz inequality, we have

$$
a a_{*}=\sum_{x, y} A(x) A_{*}(y) S(x+y) \leq d^{1 / 2} \mathrm{E}^{1 / 4}(A) \mathrm{E}^{1 / 4}\left(A_{*}\right) .
$$

Let us begin with (23) . One can assume that $\mathrm{E}(A) \geq d^{-1} a^{2} a_{*}^{2}$, the opposite case is similar. By Lemma 7, we get

$$
\frac{\mathrm{E}_{3 / 2}(A)}{a} \geq d^{-3 / 2} c^{-1 / 2} a^{2-\alpha / 2} a_{*}^{3} .
$$

Denote by $f_{j}, \mu_{j}$ the eigenfunctions and the eigenvalues of hermitian nonnegative operator

$$
\left(\tilde{\mathrm{T}}_{A, A_{*}}^{S}\left(\tilde{\mathrm{T}}_{A, A_{*}}^{S}\right)^{*}\right)(x, y)=\mathcal{C}_{3}\left(A_{*}, S, S\right)(x, y) A(x) A(y) .
$$

By Lemma 13 we know that $f_{1}(x)=A(x) / a^{1 / 2}$ and $\mu_{1}=a a_{*}$. Using the lemma again as well as bound (28), we obtain

$$
\begin{gathered}
\sigma:=\sum_{x, y \in A} \mathrm{~T}_{A}^{(A \circ A)^{1 / 2}}(x, y) \mathcal{C}_{3}\left(A_{*}, S, S\right)(x, y)=\sum_{j=1}^{a} \mu_{j}\left\langle\mathrm{~T}_{A}^{(A \circ A)^{1 / 2}} f_{j}, f_{j}\right\rangle= \\
=a^{-1} \mu_{1}\left\langle\mathrm{~T}_{A}^{(A \circ A)^{1 / 2}} A, A\right\rangle \geq d^{-3 / 2} c^{-1 / 2} a^{3-\alpha / 2} a_{*}^{4} .
\end{gathered}
$$

On the other hand, we have as in (21), (22) that

$$
\sigma^{2} \leq \mathrm{E}_{3}\left(A_{*}, A, A\right) \mathrm{E}(A, S) \leq \mathrm{E}_{3}\left(A_{*}, A, A\right)\left(c a d^{\alpha+1}\right)^{1 / 2} .
$$

Using calculations similar to Lemma 7, one can show that

$$
\mathrm{E}_{3}\left(A_{*}, A, A\right) \ll\left(c_{*} c^{2}\right)^{1 / 3}\left(a_{*} a^{2}\right)^{\alpha / 3} L .
$$

Substituting (31) into (30) and combining the result with (29), we obtain

$$
d^{-3} c^{-1} a^{6-\alpha} a_{*}^{8} \leq\left(c a d^{\alpha+1}\right)^{1 / 2}\left(c_{*} c^{2}\right)^{1 / 3}\left(a_{*} a^{2}\right)^{\alpha / 3} L .
$$

After some calculations, we have

$$
d \gg L^{-\frac{2}{7+\alpha}} c_{*}^{-\frac{2}{3(7+\alpha)}} c^{-\frac{13}{3(7+\alpha)}} a_{*}^{\frac{2(24-\alpha)}{3(7+\alpha)}} a^{\frac{33-10 \alpha}{3(7+\alpha)}}
$$

as required.

To prove (24), returning to (27) and applying Lemma 8, we obtain

$$
\left(a^{2} a_{*}^{2} d^{-1}\right)^{2 \alpha-1} \leq \mathrm{E}^{2 \alpha-1}\left(A_{*}, A\right) \ll_{(\alpha-1)^{-1}}\left(\sum_{x}\left(A_{*} \circ A_{*}\right)^{1 / 2}(x)(A \circ A)(x)\right)^{2 \alpha-2} \cdot c^{1 / 3} c_{*}^{\alpha / 3} a^{2 / 3} a_{*}^{\alpha^{2} / 3} .
$$

Thus

$$
a^{-1}\left\langle\mathrm{~T}_{A}^{\left(A_{*} \circ A_{*}\right)^{1 / 2}} A, A\right\rangle \gg_{(\alpha-1)^{-1}} a^{\frac{3 \alpha-1}{3(\alpha-1)}} a_{*}^{\frac{12 \alpha-6-\alpha^{2}}{6(\alpha-1)}} d^{\frac{1-2 \alpha}{2(\alpha-1)}} c^{-\frac{1}{6(\alpha-1)}} c_{*}^{-\frac{\alpha}{6(\alpha-1)}}
$$


After that use previous arguments replacing $\mathrm{T}_{A}^{(A \circ A)^{1 / 2}}$ onto $\mathrm{T}_{A}^{\left(A_{*} \circ A_{*}\right)^{1 / 2}}$. By (편), we have

$$
\begin{gathered}
a^{\frac{12 \alpha-8}{3(\alpha-1)}} a_{*}^{\frac{18 \alpha-12-\alpha^{2}}{3(\alpha-1)}} d^{\frac{1-2 \alpha}{\alpha-1}} c^{-\frac{1}{3(\alpha-1)}} c_{*}^{-\frac{\alpha}{3(\alpha-1)}} \leq\left(\mu_{1} a^{-1}\left\langle\mathrm{~T}_{A}^{\left(A_{*} \circ A_{*}\right)^{1 / 2}} A, A\right\rangle\right)^{2} \leq \mathrm{E}_{3}\left(A_{*}, A, A\right) \mathrm{E}\left(A_{*}, S\right) \leq \\
\leq\left(c_{*} a_{*} d^{\alpha+1}\right)^{1 / 2}\left(c_{*} c^{2}\right)^{1 / 3}\left(a_{*} a^{2}\right)^{\alpha / 3} L .
\end{gathered}
$$

After some calculations, we get the required bound.

Finally, to get (26) just apply the arguments above to get

$$
a_{*}^{2} \mathrm{E}_{3 / 2}^{2} \leq\left(c a d^{\alpha+1}\right)^{1 / 2}\left(c_{*} c^{2}\right)^{1 / 3}\left(a_{*} a^{2}\right)^{\alpha / 3} L
$$

and use the lower bound $\mathrm{E}_{3 / 2}^{2}(A) \geq a^{6} /|A-A|$. Of course, one can replace $A$ to $A_{*}$ in (26) and vice versa. This completes the proof.

Theorem above gives us a consequence to sumsets/difference sets for convex sets.

Corollary 15 Let $A, A_{*} \subset \mathbb{R}$ be two convex sets. Then

$$
\left|A \pm A_{*}\right| \gg \max \left\{|A|^{\frac{8}{9}}\left|A_{*}\right|^{\frac{2}{3}},\left|A_{*}\right|^{\frac{8}{9}}|A|^{\frac{2}{3}}\right\} \cdot \log ^{-\frac{2}{9}}\left(|A|\left|A_{*}\right|\right) .
$$

In another corollary we obtain Theorem 1.3 from [9] as well as Corollary 1.4 from the paper. These results can be considered as theorems on lower bounds for sums of SzT-type sets of special form.

Corollary 16 We have

$$
|A+f(A)| \gg \frac{|A|^{24 / 19}}{(\log |A|)^{2 / 19}}
$$

for any strictly convex or concave function $f$. Further

$$
|A A|^{6}|A-A|^{5} \gg \frac{|A|^{14}}{\log ^{2}|A|}
$$

In particular

$$
\max \{|A A|,|A-A|\} \gg|A|^{14 / 11} \log ^{-2 / 11}|A|
$$

Proof. Indeed, to obtain (33) just apply (25) with $A=A, A_{*}=f(A)$ and $c(A)=c_{*}(A)=$ $|f(A)+A|^{2}|A|^{-1}$. To get (34), we use (26) with $A=A_{*}$ having

$$
|A-A|^{5 / 2} \gg|A|^{11 / 2} c^{-3 / 2}(A) \log ^{-1}|A| .
$$

After that recall $c(A)=M^{2}|A|$ with $M=|A A| /|A|$. This concludes the proof. 


\section{References}

[1] M. Z. Garaev, On lower bounds for $L_{1}-$ norm of exponential sums, Mathematical Notes 68 (2000), 713-720.

[2] M. Z. Garaev, K-L. Kueh, On cardinality of sumsets, J. Aust. Math. Soc. 78 (2005), $221-224$.

[3] G. Elekes, M. Nathanson, I. Ruzsa, Convexity and sumsets, Journal of Number Theory, 83:194-201, 1999.

[4] N. Hegyvári, On consecutive sums in sequences, Acta Math. Acad. Sci. Hungar. 48 (1986), $193-200$.

[5] A. Iosevich, V. S. Konyagin, M. Rudnev, V. Ten, On combinatorial complexity of convex sequences, Discrete Comput. Geom. 35 (2006), 143-158.

[6] T. G. F. Jones, O. Roche-Newton, Improved bounds on the set $A(A+1)$, J. Combin. Theory Ser. A 120 (2013), no. 3, 515-526.

[7] S.V. Konyagin, M. Rudnev, On new sum-product type estimates, SIAM J. Discrete Math. 27 (2013), no. 2, 973-990.

[8] L. LI, On a theorem of Schoen and Shkredov on sumsets of convex sets, arXiv:1108.4382v1 [math.CO].

[9] L. Li, O. Roche-Newton, Convexity and a sum-product type estimate, Acta Arith. 156 (2012), no. 3, 247-255.

[10] B. Murphy, O. Roche-Newton, I. D. Shkredov, Variations on the sum-product problem, arXiv:1312.6438v2 [math.CO] 8 Jan 2014.

[11] T. Schoen, On convolutions of convex sets and related problems, preprint.

[12] T. Schoen, I.D. Shkredov, Higher moments of convolutions, J. Number Theory 133 (2013), no. 5, 1693-1737.

[13] T. Schoen, I. D. Shkredov, On sumsets of convex sets, Comb. Probab. Comput. 20 (2011), 793-798.

[14] I.D. Shkredov, Some new inequalities in additive combinatorics, Moscow J. Combin. Number Theory 3 (2013), 237-288.

[15] I.D. Shkredov, Some new results on higher energies, Transactions of MMS, 74:1 (2013), 35-73.

[16] J. Solymosi, Sumas contra productos, Gaceta de la Real Sociedad Matematica Espanola, ISSN 1138-8927, 12 (2009).

[17] T. TaO, V. Vu, Additive Combinatorics, Cambridge University Press (2006). 
I.D. Shkredov

Steklov Mathematical Institute,

ul. Gubkina, 8, Moscow, Russia, 119991

and

IITP RAS,

Bolshoy Karetny per. 19, Moscow, Russia, 127994

ilya.shkredov@gmail.com 